\title{
MICA/B and ULBP1 NKG2D ligands are independent predictors of good prognosis in cervical cancer
}

Hanbyoul Cho 1,2,3, Joon-Yong Chung ${ }^{3}$, Sunghoon Kim²,4, Till Braunschweig ${ }^{3,5}$, Tae Heung Kang ${ }^{6}$, Jennie Kim³, Eun Joo Chung ${ }^{7}$, Stephen M Hewitt ${ }^{3^{*}}$ and Jae-Hoon Kim ${ }^{1,2^{*}}$

\begin{abstract}
Background: NKG2D (natural killer group 2, member D) is thought to play an important role in mediating the activation of anticancer immune response. Expression of NKG2D ligands (NKG2DLs) is pronounced in malignancies and the heterogeneity of NKG2DL expression remains unclear. Here, we investigate the expression and clinical significance of NKG2DLs in cervical cancer.

Methods: Immunohistochemical analyses of MICA/B, ULBP1, ULBP2, ULBP3, RAET1E, and RAET1G were performed using tissue microarray analysis of 200 cervical cancers, 327 high-grade cervical intraepithelial neoplasias (CINs), 99 low-grade CINs, and 541 matched nonadjacent normal cervical epithelial tissues and compared the data with clinicopathologic variables, including the survival of cervical cancer patients.

Results: MICA/B, ULBP1, and RAET1E expression was higher in cervical cancer than in low-grade CIN $(p<0.001$, $p=0.012, p=0.013$, respectively) and normal cervix (all $p<0.001$ ). Among these markers, expression of ULBP1 was significantly different depending on patient tumor stage $(p=0.010)$ and tumor size $(p=0.045)$. ULBP1 expression was correlated with MICA/B $(p<0.001)$ and ULBP2 $(p=0.002)$ expression in cervical cancer. While MICA/B+ or ULBP1+ patients had improved disease-free survival time $(p=0.027$ and $p=0.009$, respectively) relative to that of the low expression group, RAET1E+ or RAET1G + was correlated with shorter survival time $(p=0.018$ and $p=0.029$, respectively). However, in terms of overall survival, the ULBP1+ group had significantly longer survival time than the low expression group $(p=0.009)$. Multivariate analysis indicated that MICA/B+/ULBP1+ $(H R=0.16, p=0.015)$ and ULBP1+ (HR $=0.31, p=0.024)$ are independent prognostic factors of disease-free survival in cervical cancer.
\end{abstract}

Conclusions: High expression of either ULBP1 or MICA/B and ULBP1 combined is an indicator of good prognosis in cervical cancer, suggesting their potential utility as prognostic tests in clinical assessment.

Keywords: Cervical cancer, Tissue microarray, Immunohistochemistry, NKG2D ligands

\section{Background}

Cervical cancer is the second most common malignant tumor affecting women worldwide, causing an estimated 273,200 deaths annually, and is the most common tumor in developing countries [1]. Persistent infection with one of the high-risk forms of human papillomavirus (HPV;

\footnotetext{
* Correspondence: genejock@helix.nih.gov; jaehoonkim@yuhs.ac

${ }^{3}$ Tissue Array Research Program, Laboratory of Pathology, National Cancer Institute, National Institutes of Health, 10 Center Drive, MSC 1500, Bethesda, MD 20892, USA

'Department of Obstetrics and Gynecology, Gangnam Severance Hospital, Yonsei University College of Medicine, 146-92 Dogok-Dong, Gangnam-Gu, Seoul 135-720, Korea

Full list of author information is available at the end of the article
}

types 16 and 18) has been shown to be a major etiological factor of HPV-related premalignant lesions and cervical cancer [2]. Although the vast majority of cervical cancers are derived from cervical intraepithelial neoplasia (CIN), the majority of genital HPV infections are clinically undetectable and clear in 10-16 months; only a very small proportion progress into an invasive cervical cancer. It is well known that persistent HPV infection causes progression from low-grade CIN to high-grade CIN, and eventually to a malignant cervical cancer in a multistep process [3-5]. The progression of these lesions may be due to an adverse tumor environment, wherein the mucosal 
immune response may be unable to completely remove malignant cells.

Host immune response to HPV appears to be critical in determining the outcome of infection. For example, among immunocompromised women, HPV infection is detected more frequently, the incidence of CIN is higher, and the risk of CIN recurrence after treatment is higher [6]. Innate immune response is thought to be the first line of defense at mucosal surfaces. Natural killer (NK) cells are important cytolytic and cytokine-producing effector cells of the innate immune system. These cells have the ability to attack tumor cells and cells infected with viruses and some bacteria without presentation of tumorspecific antigens [7]. Furthermore, intratumoral NK cell accumulation has been correlated with improved survival rates in patients with various solid tumors $[8,9]$. In the case of cervical cancer, Garzetti et al. reported that NK cell activity was related to prognostic parameters and clinical outcome [10].

NKG2D (natural killer group 2, member D) is a C-type lectin-like activating receptor expressed on the surface of NK cells and a variety of $\mathrm{T}$ cell subsets including CD8+ cytotoxic T cells [11]. Human NKG2D ligands (NKG2DLs) consist of two members of the MHC class I-related chain (MIC) family (MICA and MICB) and six members of the UL16 binding protein or retinoic acid early transcript (ULBP/RAET) family (ULBP1, ULBP2, ULBP3, RAET1E, RAET1G, and RAET1L) [12]. NKG2DL expression is highly restricted in healthy tissues, but can be stimulated by multiple stimuli, including infection and heat shock, and by cellular transformation [12]. It is also broadly expressed in a variety of tumors, including hematologic and epithelial malignancies [12,13], cervical cancers [14], and cancer cell lines [15,16]. The mechanisms regulating NKG2DL expression in carcinogenesis still remain to be elucidated, although activation of DNA damage response pathways and expression of the BCR/ABL oncogene have been implicated [16-18].

In the current study, we hypothesized that the expression of some or all types of NKG2DLs in cervical neoplasias are correlated with tumor progression. To explore this hypothesis, we investigated the expression of MICA/B, ULBP1, ULBP2, ULBP3, RAET1E, and RAET1G in normal cervical epithelium and cervical neoplasia in a large series of formalin-fixed, paraffin-embedded tumor samples made by using high-throughput tissue microarray (TMA) technology. Because HPV is associated with cervical carcinogenesis, we also examined the relationship between HPV status and NKG2DL expression in cervical neoplasia.

\section{Methods}

\section{Patients and tumor samples}

The study subjects were comprised of 200 cervical cancer and 426 cervical intraepithelial neoplasias (CINs) patients who underwent surgical resection at Gangnam Severance Hospital, Yonsei University College of Medicine between March 1996 and March 2010. Additional paraffin blocks were provided by the Korea Gynecologic Cancer Bank through the Bio \& Medical Technology Development Program of the Ministry of Education, Science and Technology, Korea. All patients had a histological diagnosis of cervical carcinoma or CIN, and the cervical cancer patients were clinically staged according to the International Federation of Gynecology and Obstetrics (FIGO) staging system. Patients with cervical cancer underwent type 3 radical hysterectomy with pelvic lymph node dissection, and, in cases of increased risk of relapse (assessed from spread to lymph node, parametrial invasion, and cancer close to resection margins), platinum-based concurrent chemoradiation was added. Medical records were reviewed to obtain data including age, Hybrid Capture 2 results, surgical procedure, survival time, and survival status. Response to therapy was assessed by either computed tomography or magnetic resonance imaging in accordance with the Response Evaluation Criteria in Solid Tumors (RECIST, version 1.0) [17]. Data on tumor size, cell type, tumor grade, and lymph node metastases were obtained from pathology reports. Tissue samples were collected from patients who had signed informed consent forms, which was approved by the Institutional Review Boards of Gangnam Severance Hospital. This study was additionally approved by the Office of Human Subjects Research at the National Institute of Health.

\section{Tissue microarray construction}

Tissue cores, from formalin-fixed, paraffin-embedded tissue blocks obtained from 626 patients with primary invasive cervical cancer or CIN and 541 matched nonadjacent normal cervical epithelia were arrayed into a recipient paraffin block with a manual tissue arrayer MTA-1 (Beecher Instruments Inc., Silver Spring, MD). These normal cervical tissue cores were obtained from the same block at locations distant from the cancer cells or from different block that contained enough normal epithelial cells for further IHC analyses. For each case, a representative tumor area was carefully selected from a hematoxylin and eosin (H\&E)-stained section of the donor block. Four 1.0-mm-diameter cores consisting of matched tumor specimen and normal epithelial samples were retrieved from selected regions of a patient's donor block. The presence of tumor tissues on the tissue microarray (TMA) was verified with H\&E staining. At every 50 th section, multiple 5 - $\mu$ m-thick sections were cut with a microtome and H\&E staining of TMA slides were examined for the presence of tumor cells.

\section{Cell culture}

Human cervical cancer cell lines were obtained from two sources: C-33A, CaSki, HeLa, ME-180 and SiHa from 
American Type Culture Collection (ATCC, Manassas, VA) and SUN-17 from Korean Cell Line Bank (KCLB, Seoul, Korea). CaSki, HeLa, ME-180, and SUN-17 cells were cultured in vitro in RPMI 1640 while C-33A and SiHa cells were cultured in DMEM (Dulbecco's modified Eagle's medium). Both media were supplemented with $10 \%$ fetal bovine serum, 50 units/ml of penicillin/ streptomycin, $2 \mathrm{mM}$ L-glutamine, $1 \mathrm{mM}$ sodium pyruvate, and $2 \mathrm{mM}$ non-essential amino acids, and cells were grown at $37^{\circ} \mathrm{C}$ with $5 \% \mathrm{CO}_{2}$.

\section{Flow cytometry analysis}

For in vitro flow cytometry analysis, $2 \times 10^{5}$ tumor cells were incubated with $0.5 \mu \mathrm{g}$ recombinant human NKG2D Fc chimera ( $\mathrm{R} \& \mathrm{D}$ systems, Minneapolis, MN) and then PE-conjugated anti-human Fc secondary antibody was used as a detection antibody (BD Bioscience, San Jose, CA). CELLQuest software (Becton Dickinson Immunocytometry System, Mountain View, CA) was used for FACScan analysis.

\section{Immunohistochemistry}

Immunohistochemical staining of MICA/MICB, ULBP1, ULBP2, ULBP3, RAET1E, and RAET1G was performed by using streptavidin-biotin peroxidase method. Prior to applying IHC with TMA section, we tested whole section for immunohistochemial staining condition. Briefly, the TMA sections were deparaffinized by xylene and then rehydrated through a descending alcohol gradient. Endogenous peroxidase activity was blocked by $10 \mathrm{~min}$ of incubation in $3 \% \mathrm{H}_{2} \mathrm{O}_{2}$. To retrieve antigenicity, sections were immersed in antigen retrieval buffer, pH 9 (Dako, Carpinteria, CA), and heated for $20 \mathrm{~min}$ in a steam pressure cooker (Pascal, Dako). Slides for ULBP1 and ULBP2, however, were heated for $10 \mathrm{~min}$ instead of $20 \mathrm{~min}$ at high pressure. To block nonspecific staining, sections were treated with Protein Block (Dako) for $20 \mathrm{~min}$. The sections were incubated with anti-MICA/ MICB antibody (Novus Biologicals, Littleton, CO; mouse monoclonal antibody, Clone 6D4, 1:50 for $120 \mathrm{~min}$ ), anti-ULBP1 antibody (Sigma-Aldrich, St. Louis, MO; rabbit polyclonal antibody, Cat.\# HPA007547, 1:50 for $120 \mathrm{~min}$ ), anti-ULBP2 antibody (R\&D systems; goat polyclonal antibody, Cat.\# AF1298, 1:50 for $30 \mathrm{~min}$ ), anti-ULBP3 antibody (Abcam, Cambridge, MA; mouse monoclonal antibody, Clone MM0594-6E12, 1:500 for 120 min), anti-RAET1E antibody (Novus Biologicals; mouse polyclonal antibody, Cat.\# H00135250-B01, 1:2000 overnight at $4^{\circ} \mathrm{C}$ ), and anti-RAET1G antibody (Novus Biologicals; mouse polyclonal antibody, Cat.\# H00353091B01, 1:1000 overnight at $4^{\circ} \mathrm{C}$ ) in a Dako autostainer plus (Dako). Different systems were used for the detection of primary antibodies: Dako EnVision + Dual Link System-HRP (Dako) for MICA/MICB and ULBP3; Dako
EnVision FLEX+ (Dako) for ULBP1, RAET1E, and RAET1G; and Dako LSAB 2 System-HRP (Dako) for ULBP2. The stain was visualized using $\mathrm{DAB}^{+}$kit $(3,3$ 'Diaminobenzidine; Dako) and then lightly counterstained with hematoxylin. The slides were covered and observed under a light microscope (Axioplot, Carl Zeiss, Jena, Germany). Negative controls were processed by omitting the primary antibodies, and TMAs included colorectal cancer positive control tissues [19].

\section{Evaluation of IHC staining}

For the assessment of NKG2DL staining, two scores were assigned to each core: (a) the staining intensity (no evidence of staining, 0; weak staining, 1+; moderate staining, 2+; and strong positive staining in most cells, $3+$ ) and (b) the percentage of positively stained epithelial cells (no cells staining positive, 0 ; less than $25 \%$ of cells staining positive, $1+; 25-50 \%$ of cells staining positive, $2+; 50-75 \%$ of cells staining positive, $3+$; and more than $75 \%$ of cells staining positive, $4+$ ). An overall protein expression score was calculated by multiplying the intensity and positivity scores (overall score range, $0-12$ ). The IHC staining score was then dichotomized into low expression ( $\leq$ mean score of cancer specimens) and high expression (> mean score of cancer specimens). Slides were scored without any clinical information, and the final immunostaining score reported was the average of two independent pathologists, both with experience in the analysis of tissue microarray.

\section{Statistical analysis}

Statistical analyses were performed using SPSS version 18.0 (SPSS Inc., Chicago, IL). The statistical significance of the differences in staining score of NKG2DLs in the different groups was calculated by the Mann-Whitney test and the Kruskal-Wallis test. Overall and disease-free survival curves were generated by the Kaplan-Meier method and the difference between the survival curves was calculated by the log-rank test. The Cox proportional hazards model was used for multivariate analysis to determine independent significance of relevant clinical covariates. In all cases, a $p$ value $<0.05$ was considered statistically significant.

\section{Results}

\section{Clinicopathologic characteristics of patient cohort}

Table 1 summarizes patient clinicopathologic characteristics. The overall mean age of patients was $40.6 \pm 9.8$ years for low-grade CIN, $38.9 \pm 11.2$ years for high-grade CIN, and $49.4 \pm 11.7$ years for cervical cancer. The distribution of FIGO staging for the 200 cases of cervical cancer is as follows: 138 stage I, 53 stage II, and 9 stage IV. The following cell types were assigned according to World Health Organization (WHO) criteria: 164 squamous cell carcinomas, 30 adenocarcinomas/adenosquamous carcinomas, 4 
Table 1 Characteristics of patients

\begin{tabular}{|c|c|c|}
\hline Variable & Number & $\%$ \\
\hline Age (years) & $42.58 \pm 12.15^{\mathrm{a}}$ & \\
\hline \multicolumn{3}{|c|}{ Diagnostic category } \\
\hline Normal & 541 & 46.4 \\
\hline Low-grade CIN & 99 & 8.5 \\
\hline High-grade CIN & 327 & 28.0 \\
\hline Cancer & 200 & 17.1 \\
\hline \multicolumn{3}{|l|}{ FIGO stage } \\
\hline । & 138 & 69.0 \\
\hline$\|$ & 53 & 26.5 \\
\hline IV & 9 & 4.5 \\
\hline \multicolumn{3}{|l|}{ Tumor grade $^{b}$} \\
\hline Well/Moderate & 117 & 65.7 \\
\hline Poor & 61 & 34.3 \\
\hline \multicolumn{3}{|l|}{ Cell type } \\
\hline SCC & 164 & 82.0 \\
\hline AC/ASC & 30 & 15.0 \\
\hline Others & 6 & 3.0 \\
\hline \multicolumn{3}{|l|}{ Tumor size } \\
\hline$\leq 4 \mathrm{~cm}$ & 145 & 72.5 \\
\hline$>4 \mathrm{~cm}$ & 55 & 27.5 \\
\hline \multicolumn{3}{|c|}{ Lymphovascular invasion ${ }^{c}$} \\
\hline Negative & 95 & 64.6 \\
\hline Positive & 52 & 35.4 \\
\hline \multicolumn{3}{|c|}{ Lymph node metastasis $^{d}$} \\
\hline Negative & 141 & 77.5 \\
\hline Positive & 41 & 22.5 \\
\hline \multicolumn{3}{|c|}{ Chemoradiation response ${ }^{e}$} \\
\hline Good & 38 & 77.6 \\
\hline Bad & 11 & 22.4 \\
\hline \multicolumn{3}{|l|}{ SCC antigen ${ }^{f}$} \\
\hline Negative & 100 & 66.7 \\
\hline Positive & 50 & 33.3 \\
\hline \multicolumn{3}{|l|}{ HPV test in $\mathrm{CIN}^{\mathrm{g}}$} \\
\hline Negative & 39 & 11.5 \\
\hline Positive & 300 & 88.5 \\
\hline
\end{tabular}

CIN, cervical intraepithelial neoplasia; FIGO, International Federation of Gynecology and Obstetrics; SCC, squamous cell carcinoma; AC, adenocarcinoma; ASC, adenosquamous carcinoma. ${ }^{a}$ mean \pm standard deviation, ${ }^{\mathrm{b}}$ calculated only for the 178 cases with available information on tumor grade, 'calculated only for the 147 cases with available information on examined lymphovascular invasion, ${ }^{d}$ calculated only for the 182 cases with

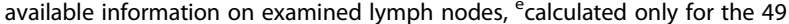
cases with available information on chemoradiation response, ${ }^{f}$ calculated only for the 114 cases with available information on SCC antigen level, ${ }^{9}$ calculated only for the $339 \mathrm{CIN}$ cases with available information on HPV infection. small cell carcinomas, 1 neuroendocrine, and 1 clear cell carcinomas. HC2-confirmed HPV infection rate was 78.7\% (74/94) in low-grade CIN, $92.2 \%(226 / 245)$ in high-grade $\mathrm{CIN}$, and $93.9 \%(92 / 98)$ in cervical cancer.

\section{Confirmation of NKG2DLs in cervical cancer cell lines}

Expression of NKG2DLs was determined by flow cytometric assay using recombinant human NKG2D-Fc chimera protein in cell cultures prior to IHC analysis for individual NKG2DL expression in cervical cancer tissues. NKG2D-Fc chimera protein binds to NKG2D through ligand-receptor interaction on the cell surface, and it is detected by anti-Fc antibody conjugated with fluorophores. As a result, using NKG2D-Fc chimera protein in flow cytometric assay allows us to confirm the expression and binding ability of NKG2DLs even without a characterization of ligands. As shown in Figure 1, expression of NKG2DLs was determined in six different cervical cancer cell lines (C-33A, CaSki, HeLa, ME-180, SiHa and SUN-17). Five cancer cell lines (all but C-33A) expressed NKG2DLs, which could bind to NKG2D, supporting the hypothesis that cervical cancers express NKG2DLs in vivo.

\section{Expression of individual NKG2DLs in cervical neoplasias}

We then performed IHC analysis of MICA/MICB, ULBP1, ULBP2, ULBP3, RAET1E, and RAET1G in 200 cervical cancer specimens, 327 high-grade CINs, 99 lowgrade CINs, and 541 matched nonadjacent normal cervical epithelial tissue samples. Representative immunohistochemical expression of individual NKG2DLs are presented in Figure 2. ULBP3 was expressed exclusively in the nucleus in both tumor and normal epithelial cells, while the other markers were expressed primarily in the cytoplasm, with some cases also demonstrating weak nucleus staining (Figure 2). Scoring results from the IHC analyses are summarized in Table 2. The TMA contains 200 cases of cervical cancer, however due to the complexity of sectioning and staining, between 180 and 195 samples could be interpreted for the individual marker. Invasive cervical cancer tissues had higher MICA/B, ULBP1, and RAET1E expression than CIN or normal cervical epithelial tissues (all $p<0.001$ ). This trend of progressively increasing MICA/B, ULBP1, and RAET1E expression corresponded to the phases of cervical cancer progression and was significant according to Spearman's rank correlation ( $\rho$-value of $0.313[p<0.001]$, $0.285[p<0.001]$, or $0.136[p<0.001]$, respectively). ULBP2 and ULBP3 expression, on the other hand, was higher in low-grade CIN than in normal epithelium but gradually decreased in high-grade CIN and cervical cancer $(p=0.001$ and $p=0.017$, respectively).

\section{Association of NKG2DLs}

To determine the association between expression of MICA/MICB, ULBP1, ULBP2, ULBP3, RAET1E, and 


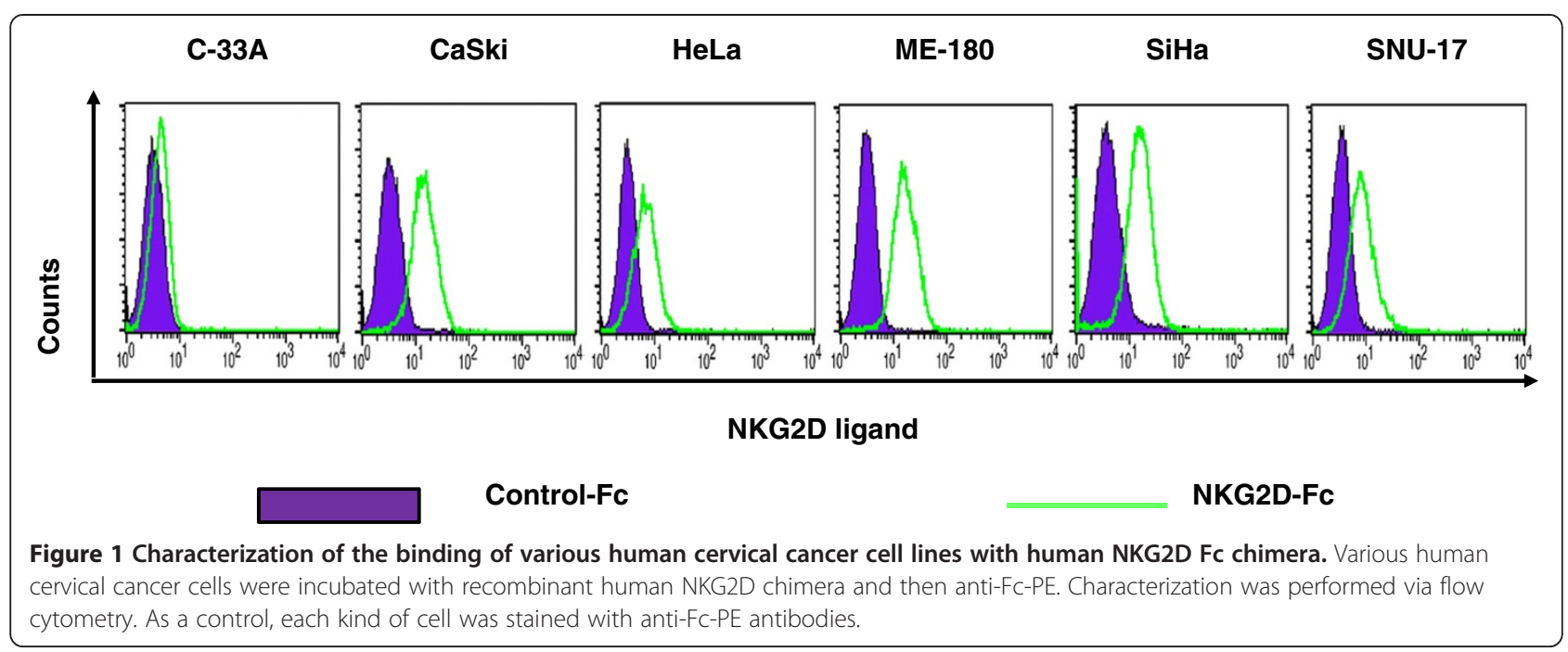

RAET1G, Spearman's rank correlation analysis was performed (Table 3). Cervical cancer lesions were evaluated for co-expression between individual NKG2DLs. MICA/B expression was significantly correlated with the expression of ULBP1 (Spearman's rho $=0.677, p<0.001$ ) or ULBP2 (Spearman's rho $=0.257, p<0.001$ ), whereas MICA/B expression was not significantly correlated with those of ULBP3 (Spearman's rho $=0.119, p=0.112$ ), RAET1E (Spearman's rho $=0.044, p=0.555$ ), or RAET1G (Spearman's rho $=-0.042, p=0.574$ ).

\section{Prognostic significance of NKG2DL expression}

Finally, to investigate the prognostic significance of the expression of individual NKG2DLs in cervical cancer, we studied the correlation of NKG2DL expression with overall and disease-free survival. Clinicopathologic and outcome information were available for all 200 cervical cancer patients who were monitored for survival and recurrence. Kaplan-Meier plots demonstrated that patients with high MICA/B (Log-rank $p=0.027$ ) or ULBP1 (Log-rank $p=0.009$ ) expression and low RAET1E (Log-rank $p=0.018)$ or RAET1G (Log-rank $p=0.029)$ expression had significantly longer disease-free survival time (Table 4, Figure 3A and B). When analyzed individually for effect on overall survival, high ULBP1 expression predicted significantly longer survival time (Log-rank $p=0.007$ ) (Table 4 , Figure $3 \mathrm{D}$ and E). In particular, when survival of patients with expression of high MICA/B/high ULBP1 was compared with those of other patients, Kaplan-Meier analysis revealed a significant difference in both disease-free $(p<0.001$, Figure $3 C)$ and overall survival ( $p=0.001$, Figure $3 \mathrm{~F})$. A Cox multivariate proportional hazards analysis showed that advanced stage (hazard ratio $=3.60[95 \% \mathrm{CI}, 1.36-9.55], \quad P=0.010$ ) and lymph node metastasis (hazard ratio $=2.71[95 \%$ CI, 1.08-6.79], $p=0.032$ ) were related to poor disease-free survival, whereas high ULBP1 (hazard ratio $=0.31[95 \%$ CI, 0.11-0.86], $p=0.024$ ) and high MICA/B/high ULBP1 (hazard ratio $=0.16$ [95\% CI, 0.13-0.70], $p=0.015$ ) expression was related to good disease-free survival (Table 2). When analyzed for effect on overall survival, advanced stage (hazard ratio $=2.77$ [95\% CI, 1.13-7.76], $p=0.025$ ) and high ULBP1 (hazard ratio $=0.27$ [95\% CI, 0.07-0.97], $p=0.044$ ) expression were significant independent prognostic factors in multivariate analysis.

\section{Discussion}

Studies based on various in vivo models suggest that the immune system not only protects the host from early-stage tumors, but also promotes tumor growth through a process described as immunoediting, immunosculpting or cancer immune system, which can result in the outgrowth of more aggressive tumor cells via exposure to immune effectors and loss of immunogenicity [18,20,21]. In addition, these in vivo cancer models also strongly suggest that the activating immune receptor NKG2D stimulates anti-cancer immune responses [22-24]. Considering the fact that many human primary tumors and tumor-derived cell lines express NKG2DLs [12,16], a great deal of research is currently focused on investigating the role of NKG2D in host-mediated tumor immunity. Despite recent progress, the biological functions of NKG2DLs are not yet fully understood, and the clinicopathologic significance of these ligands in cervical cancer has yet to be reported.

In the present study, we investigated NKG2DL expression and further explored the clinical significance of NKG2DLs by using samples from a large cohort of patients including cervical cancer, precursor and corresponding normal specimens. Immunohistochemistry analysis revealed that MICA/B and ULBP1 were significantly upregulated in cervical cancer tissues compared to their corresponding normal tissues. Notably, higher MICA/B and ULBP1 


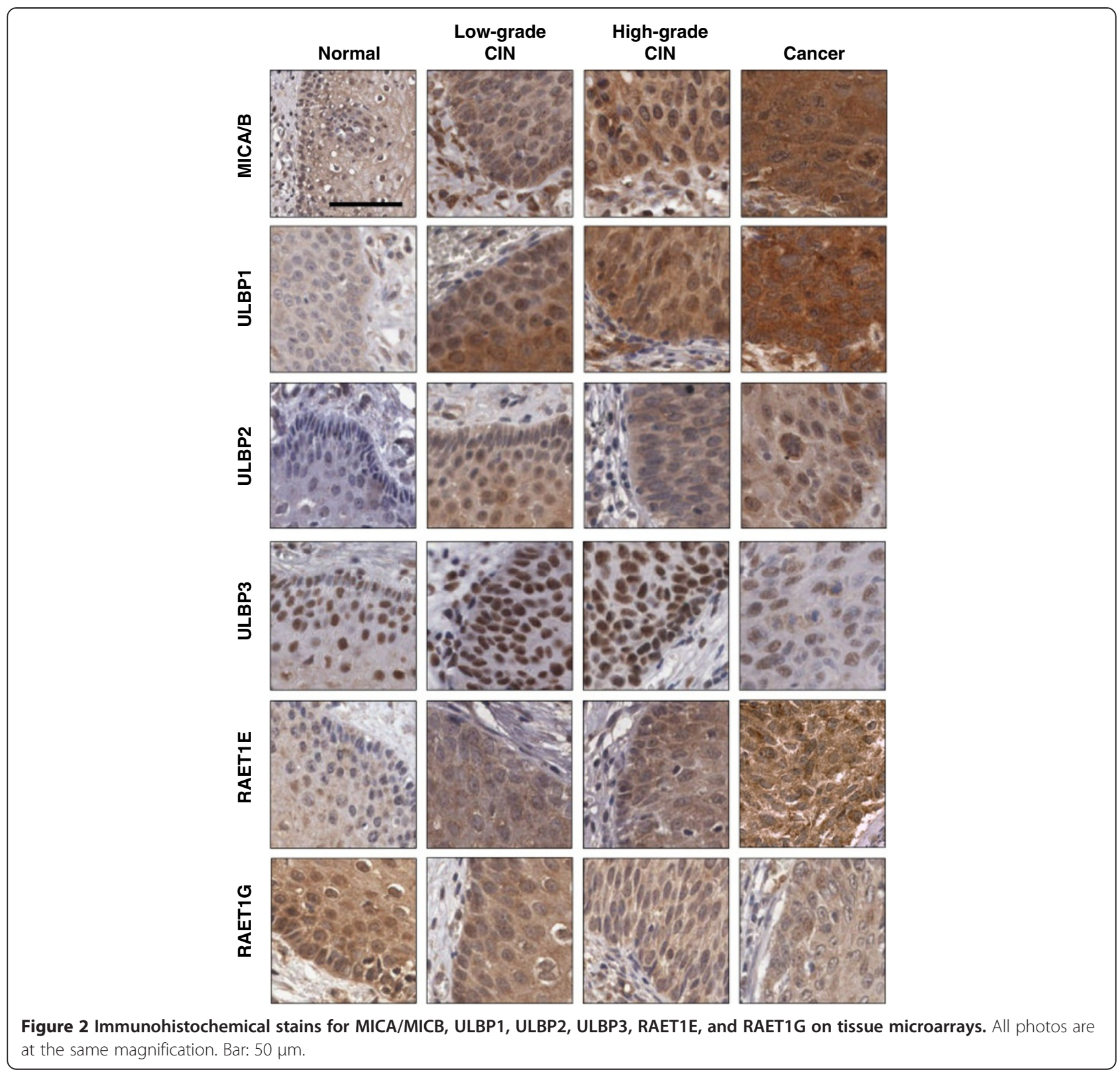

expression correlated with more advanced stages of cervical carcinogenesis, increasing from normal cervical tissue to progressively advanced stages of cervical cancer precursors (low-grade and high-grade CIN) and ultimately to invasive cancer. These findings suggest that MICA/B and ULBP1 upregulation follows malignant transformation in cervical carcinogenesis. Thus, not only can these proteins be used as potential markers in treatment and surveillance of cervical cancer, but they may also have some utility in screening. Since their expression increases even in early stages of cervical cancer, MICA/B and ULBP1 expression can be used to identify precursor lesions (i.e., CIN) that are at high risk of developing invasive cervical cancer.
In addition to the correlations between NKG2DL expression levels and stages of cervical carcinogenesis, NKG2DL expression was heterogeneous in primary cancers, as not all of the ligands were highly expressed in the same tumor. More specifically, MICA/B, ULBP1 and RAET1E expression was significantly increased in cervical cancer tissues, while ULBP2 and ULBP3 expression were higher in low-grade CIN tissues, but lower in high-grade CIN and cervical cancer tissues. Similar to our study, Textor et al. demonstrated that NKG2DL MICA expression was upregulated in squamous cervical carcinoma tissues $(n=15)$ compared to CIN $(n=28)$ and normal ectocervical tissues $(n=10)$ while ULBP2 was strongly expressed in normal ectocervical tissues [14]. Although the mechanism 
Table 2 IHC scores of NKG2DLs MICA/B, ULBP1, ULBP2, ULBP3, RAET1E, and RAET1G in 1,167 TMA specimens

\begin{tabular}{|c|c|c|c|c|c|c|c|}
\hline & & \multirow[t]{2}{*}{ Number } & \multirow[t]{2}{*}{$\%$} & \multirow{2}{*}{$\begin{array}{l}\text { Mean } \\
\text { score }\end{array}$} & \multicolumn{2}{|l|}{$95 \% \mathrm{Cl}$} & \multirow[t]{2}{*}{$p$ value } \\
\hline & & & & & Lower & Upper & \\
\hline \multirow[t]{5}{*}{ MICA/B } & Normal & 457 & 45.2 & 4.83 & 4.62 & 5.04 & $<0.001$ \\
\hline & Low-grade CIN & 90 & 8.9 & 4.89 & 4.53 & 5.25 & \\
\hline & High-grade CIN & 270 & 26.7 & 6.30 & 6.00 & 6.61 & \\
\hline & Cancer & 195 & 19.3 & 6.78 & 6.43 & 7.13 & \\
\hline & Total & 1012 & 100.0 & 5.60 & 5.45 & 5.76 & \\
\hline \multirow[t]{5}{*}{ ULBP1 } & Normal & 500 & 45.9 & 4.82 & 4.64 & 5.00 & $<0.001$ \\
\hline & Low-grade CIN & 92 & 8.4 & 5.54 & 5.14 & 5.94 & \\
\hline & High-grade CIN & 314 & 28.8 & 6.05 & 5.78 & 6.32 & \\
\hline & Cancer & 183 & 16.8 & 6.66 & 6.27 & 7.04 & \\
\hline & Total & 1089 & 100.0 & 5.54 & 5.40 & 5.68 & \\
\hline \multirow[t]{5}{*}{ ULBP2 } & Normal & 458 & 43.5 & 3.47 & 3.30 & 3.65 & 0.001 \\
\hline & Low-grade CIN & 93 & 8.8 & 4.23 & 3.82 & 4.63 & \\
\hline & High-grade CIN & 318 & 30.2 & 3.94 & 3.73 & 4.16 & \\
\hline & Cancer & 184 & 17.5 & 3.71 & 3.40 & 4.02 & \\
\hline & Total & 1053 & 100.0 & 3.72 & 3.60 & 3.84 & \\
\hline \multirow[t]{5}{*}{ ULBP3 } & Normal & 455 & 44.0 & 5.22 & 5.02 & 5.41 & 0.017 \\
\hline & Low-grade CIN & 93 & 9.0 & 5.84 & 5.37 & 6.31 & \\
\hline & High-grade CIN & 307 & 29.7 & 5.34 & 5.05 & 5.63 & \\
\hline & Cancer & 180 & 17.4 & 4.91 & 4.56 & 5.26 & \\
\hline & Total & 1035 & 100.0 & 5.26 & 5.11 & 5.40 & \\
\hline \multirow[t]{5}{*}{ RAET1E } & Normal & 461 & 44.4 & 3.57 & 3.47 & 3.68 & $<0.001$ \\
\hline & Low-grade CIN & 94 & 9.1 & 3.77 & 3.54 & 3.99 & \\
\hline & High-grade CIN & 302 & 29.1 & 3.76 & 3.62 & 3.91 & \\
\hline & Cancer & 181 & 17.4 & 4.38 & 4.12 & 4.65 & \\
\hline & Total & 1038 & 100.0 & 3.79 & 3.71 & 3.87 & \\
\hline \multirow[t]{5}{*}{ RAET1G } & Normal & 470 & 44.2 & 5.11 & 4.95 & 5.27 & 0.001 \\
\hline & Low-grade CIN & 95 & 8.9 & 5.17 & 4.83 & 5.50 & \\
\hline & High-grade CIN & 314 & 29.5 & 4.61 & 4.39 & 4.82 & \\
\hline & Cancer & 184 & 17.3 & 5.11 & 4.78 & 5.45 & \\
\hline & Total & 1063 & 100.0 & 4.97 & 4.85 & 5.08 & \\
\hline
\end{tabular}

for this heterogeneity in NKG2DL expression is complex and unclear at this time, it may be partially explained by the following reasons. First, this heterogeneity may be due to NKG2DLs having different promoters and NKG2D's ability to be expressed independently in response to diverse stress response pathways. Secondly, evidence of post-transcriptional regulation of NKG2DLs, including the involvement of micro-RNAs (miR-20a, miR-93, miR106b, miR-302d, miR-372, miR-373 and miR-520d), also indicates that NKG2D expression is further differentially regulated [25]. Lastly, some stimuli, such as DNA damage response, have been reported to result in expression of all NKG2DLs tested, while other stimuli have been shown to induce expression of other specific NKG2DLs
[26]. BCR/ABL, for example, regulates MICA, but not ULBP1-2 in K562 cells, while histone deacetylase inhibitor induces MICA, but not ULBP1-3 in hepatoma cells $[27,28]$.

Regardless of NKG2DL heterogeneity in expression, our prognostic model indicates that the presence of NKG2DLs is crucial for stimulating immune responses against tumors. Our survival analysis indicates that the high expression of NKG2DLs predicts a good prognosis, with the combination of ULBP1 and MICA/B predicting disease-free survival and ULBP1 independently predicting both disease-free and overall survival with statistical significance (Table 5). This is in agreement with a recent report on MIC/ULBP/RAET expression in 462 primary 
Table 3 Spearman's rank correlation coefficient in cervical cancer specimens

\begin{tabular}{|c|c|c|c|c|c|c|c|c|}
\hline & & & MICA/B & ULBP1 & ULBP2 & ULBP3 & RAET1E & RAET1G \\
\hline \multirow[t]{18}{*}{ Spearman's correlation } & $\mathrm{MICA} / \mathrm{B}$ & $\rho$ & 1.000 & .677 & .257 & .119 & .044 & -.042 \\
\hline & & p & NA & $<.001$ & $<.001$ & .112 & .555 & .574 \\
\hline & & $n$ & 195 & 183 & 181 & 179 & 181 & 182 \\
\hline & ULBP1 & $\rho$ & .677 & 1.000 & .232 & .140 & .018 & -.055 \\
\hline & & p & $<.001$ & NA & .002 & .068 & .820 & .476 \\
\hline & & $n$ & 183 & 183 & 174 & 171 & 169 & 170 \\
\hline & ULBP2 & $\rho$ & .257 & .232 & 1.000 & .333 & -.119 & -.107 \\
\hline & & $p$ & $<.001$ & .002 & NA & $<.001$ & .121 & .162 \\
\hline & & $n$ & 181 & 174 & 184 & 176 & 172 & 174 \\
\hline & ULBP3 & $\rho$ & .119 & .140 & .333 & 1.000 & .268 & .158 \\
\hline & & $p$ & .112 & .068 & $<.001$ & NA & $<.001$ & .038 \\
\hline & & $n$ & 179 & 171 & 176 & 180 & 172 & 173 \\
\hline & RAET1E & $\rho$ & .044 & .018 & -.119 & .268 & 1.000 & .616 \\
\hline & & $p$ & .555 & .820 & .121 & $<.001$ & NA & $<.001$ \\
\hline & & $n$ & 181 & 169 & 172 & 172 & 182 & 179 \\
\hline & RAET1G & $\rho$ & -.042 & -.055 & -.107 & .158 & .616 & 1.000 \\
\hline & & $p$ & .574 & .476 & .162 & .038 & $<.001$ & NA \\
\hline & & $n$ & 182 & 170 & 174 & 173 & 179 & 184 \\
\hline
\end{tabular}

NA, not applicable.

Table 4 Overall and disease-free survival analysis of NKG2DLs MICA/B, ULBP1, ULBP2, ULBP3, RAET1E, and RAET1G

\begin{tabular}{|c|c|c|c|c|c|c|}
\hline & \multicolumn{3}{|c|}{ Disease-Free Survival Time } & \multicolumn{3}{|c|}{ Overall Survival Time } \\
\hline & Mean & $95 \% \mathrm{Cl}$ & Log-rank $P$ & Mean & $95 \% \mathrm{Cl}$ & Log-rank $P$ \\
\hline \multicolumn{7}{|l|}{$\overline{M I C A} / \mathrm{B}$} \\
\hline Low $(n=112)$ & 127 & $112-141$ & 0.027 & 146 & $133-158$ & 0.181 \\
\hline High $(n=83)$ & 164 & $150-178$ & & 169 & $155-183$ & \\
\hline \multicolumn{7}{|l|}{ ULBP1 } \\
\hline Low $(n=106)$ & 125 & $110-140$ & 0.009 & 139 & $125-152$ & 0.007 \\
\hline High $(n=77)$ & 163 & $148-178$ & & 177 & $167-188$ & \\
\hline \multicolumn{7}{|l|}{ ULBP2 } \\
\hline $\operatorname{Low}(n=93)$ & 144 & $127-160$ & 0.279 & 165 & $152-178$ & 0.640 \\
\hline High $(n=91)$ & 148 & 134-163 & & 155 & $141-169$ & \\
\hline \multicolumn{7}{|l|}{ ULBP3 } \\
\hline Low $(n=79)$ & 136 & $121-151$ & 0.364 & 147 & $134-160$ & 0.707 \\
\hline $\operatorname{High}(n=101)$ & 139 & $122-157$ & & 159 & $144-173$ & \\
\hline \multicolumn{7}{|l|}{ RAET1E } \\
\hline Low $(n=123)$ & 157 & $144-170$ & 0.018 & 163 & $151-175$ & 0.935 \\
\hline High $(n=59)$ & 116 & $96-137$ & & 149 & $135-164$ & \\
\hline \multicolumn{7}{|l|}{ RAET1G } \\
\hline Low $(n=104)$ & 159 & $146-172$ & 0.029 & 166 & 154-179 & 0.234 \\
\hline High $(n=80)$ & 127 & $108-146$ & & 149 & $132-165$ & \\
\hline
\end{tabular}

$\mathrm{IHC}$ staining scores of the NKG2DLs were dichotomized into low expression ( $\leq$ mean score of cancer specimens) and high expression (> mean score of cancer specimens). 


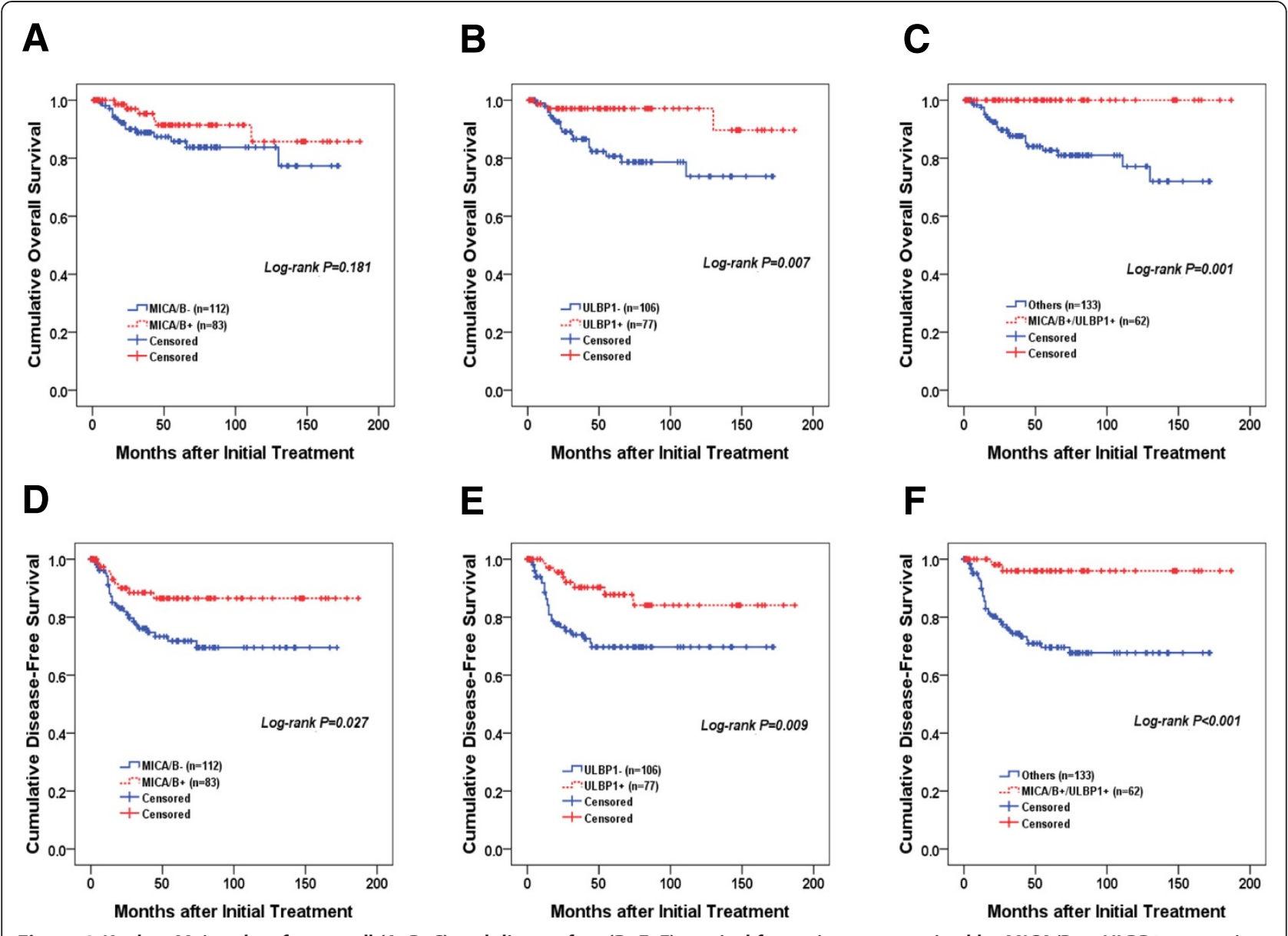

Figure 3 Kaplan-Meier plots for overall (A, B, C) and disease-free (D, E, F) survival for patients categorized by MICA/B or ULBP1 expression.

Table 5 Cox proportional univariate and multivariate analysis of the association between prognostic variables and overall and disease-free survival in cervical cancer

\begin{tabular}{|c|c|c|c|c|}
\hline & \multicolumn{2}{|c|}{ Disease-free Survival hazard ratio $[95 \% \mathrm{Cl}], P$ value } & \multicolumn{2}{|c|}{ Overall Survival hazard ratio $[95 \% \mathrm{Cl}], P$ value } \\
\hline & Univariate analysis & Multivariate analysis & Univariate analysis & Multivariate analysis \\
\hline FIGO stage & $5.32[2.75-10.30],<0.001$ & $3.60[1.36-9.55], 0.010$ & $3.97[1.65-9.54], 0.002$ & $2.77[1.13-7.76], 0.025$ \\
\hline Tumor grade (poor) & $1.65[0.85-3.22], 0.137$ & NA & $2.15[0.89-5.21], 0.087$ & NA \\
\hline Tumor size $(>4 \mathrm{~cm})$ & $2.13[1.09-4.14], 0.025$ & $1.16[0.47-2.90], 0.737$ & $1.90[0.78-4.65], 0.157$ & NA \\
\hline LN metastasis & $4.53[2.14-9.61],<0.001$ & $2.71[1.08-6.79], 0.032$ & $2.25[0.77-6.59], 0.136$ & NA \\
\hline $\mathrm{HPV}+$ & $0.68[0.08-5.21], 0.710$ & NA & $0.38[0.048-3.10], 0.370$ & NA \\
\hline MICA/B+ & $0.43[0.20-0.92], 0.032$ & $0.54[0.20-1.41], 0.213$ & $0.53[0.20-1.36], 0.189$ & NA \\
\hline ULBP1+ & $0.36[0.16-0.80], 0.012$ & $0.31[0.11-0.86], 0.024$ & $0.21[0.06-0.73], 0.014$ & $0.27[0.07-0.97], 0.044$ \\
\hline RAET1E+ & $2.17[1.12-4.2], 0.021$ & $1.90[0.84-4.32], 0.123$ & $1.03[0.41-2.60], 0.935$ & NA \\
\hline RAET1G+ & $2.09[1.05-4.15], 0.034$ & $1.62[0.68-3.87], 0.275$ & $1.69[0.70-4.09], 0.240$ & NA \\
\hline MICA/B+/ULBP1+ & $0.11[0.02-0.45], 0.002$ & $0.16[0.03-0.70], 0.015$ & $0.026[0.00-1.17], 0.060$ & NA \\
\hline
\end{tabular}

$\mathrm{IHC}$ staining scores of NKG2DLs were dichotomized into low (-) expression ( $\leq$ mean score of cancer specimens) and high (+) expression (> mean score of cancer specimens).

FIGO, International Federation of Gynecology and Obstetrics; LN, lymph node; HPV, human papilloma virus; Cl, confidence interval; NA, not applicable. 
colorectal tumors, which states that high expression of MIC or RAET1G predicts improved patient survival [19]. Considering the fact that NKG2DLs are involved in anti-cancer immune responses, an association between high expression of NKG2DLs and good prognosis is quite reasonable. Thus, when identifying patients with cervical cancer at increased risk of tumor invasion and/or progression, examining the expression levels of ULBP1 or MICA/B via IHC may have utility. Furthermore, these findings all emphasize that NKG2DLs have important functions in the biological mechanism underlying the development and/or growth of human cervical cancer.

However, in contrast to our results, recent studies by Li et al. demonstrated that high expression of MICA/B and ULBP2 is associated with poor prognosis in ovarian cancer patients [29]. This, together with our results, indicates that although NKG2DLs induce anti-cancer immune responses, the extent of these responses can markedly differ depending on the type of cancer. Such differences arise because NKG2DLs depend heavily on the tissue microenvironment where an assemblage of interactions by signals of cellular receptors and cytokines take place [30]. Since these interactions differ depending on the type of the cancer, different NKG2D responses are elicited [31,32]. Thus, not only are NKG2DLs expressed in response to different cancer-related pathways, but also their expression is highly heterogeneous.

Intriguingly, although the results of our multivariate analysis were not significant, in univariate analysis, high RAET1E or RAET1G expression was associated with poor disease-free survival (Table 5). This finding agrees partially with a recent report on NKG2DL expression in 357 ovarian cancers, wherein high expression levels of ULBP2 and RAET1G was inversely correlated to the disease survival [33]. The lack of complete agreement may be due to the use of a different cancer type. However, more importantly, it can be explained by a study performed by Cao et al., in which RAET1E was able to produce soluble protein lacking a transmembrane region that weakened NKG2D-mediated NK cell cytotoxicity to tumor cells, despite that RAET1E and RAET1G contain both transmembrane and cytoplasmic domains [34]. Given the results of Cao et al.'s study and the fact that many isoforms were also stained during RAET1E and RAET1G IHC staining, it is possible that high RAET1E and RAET1G expression may be associated with poor prognosis.

\section{Conclusions}

Overall, the expression of several NKG2DLs, namely MICA/B, ULBP1, and RAET1E, were increased in cervical cancer patients. In multivariate analysis, FIGO stage, lymph node metastasis, high ULBP1 expression and high combined MICA/B and ULBP1 expression were independent predictors of good prognosis. These findings all underscore the importance of NKG2D function in cervical tumor progression and cancer immunosurveillance, and suggest that combinatorial analysis of NKG2DL expression may assist in realizing improved prognostic classification of cervical and other carcinomas.

\section{Abbreviations}

NKG2DL: NKG2D ligand; HPV: Human papillomavirus; CIN: Cervical intraepithelial neoplasia; LSIL: Low-grade squamous intraepithelial lesions; HSIL: High-grade squamous intraepithelial lesions; NK: Natural killer; FIGO: International federation of gynecology and obstetrics; TMA: Tissue microarray; H\&E: Hematoxylin and eosin; SCC: Squamous cell carcinoma; AC: Adenocarcinoma; ASC: Adenosquamous carcinoma.

\section{Competing interests}

The authors declare that they have no competing interests.

\section{Authors' contributions}

HC, J-YC, SMH and J-HK conceived of the study and devised the experimental design. SMH and J-HK designed and build the tissue microarrays. HC, J-YC, SK, TB, THK and JK performed experiments. HC, J-YC, SK, TB, EJC, SMH and J-HK performed data analysis for experiments or clinical records. HC and J-YC drafted the final version of the manuscript and figure legends. SMH and J-HK revised the figures, added critical content to the discussion and was responsible in revising all portions of the submitted portion of the manuscript. All authors read and approved the final manuscript.

\section{Acknowledgments}

This work was supported in part by grants from the Basic Science Research Program through the National Research Foundation of Korea (NRF), funded by the Ministry of Education, Science and Technology (2011-0007146) and faculty research grants from Yonsei University College of Medicine for 2014 (6-2014-0072) and Intramural Research Program of the National Institutes of Health, National Cancer Institute, and Center for Cancer Research.

\section{Author details}

'Department of Obstetrics and Gynecology, Gangnam Severance Hospital, Yonsei University College of Medicine, 146-92 Dogok-Dong, Gangnam-Gu, Seoul 135-720, Korea. ${ }^{2}$ Institute of Women's Life Medical Science, Yonsei University College of Medicine, Seoul, Korea. ${ }^{3}$ Tissue Array Research Program, Laboratory of Pathology, National Cancer Institute, National Institutes of Health, 10 Center Drive, MSC 1500, Bethesda, MD 20892, USA. ${ }^{4}$ Department of Obstetrics and Gynecology, Severance Hospital, Yonsei University College of Medicine, Seoul, Korea. ${ }^{5}$ Institute of Pathology, RWTH Aachen University, Aachen, Germany. ${ }^{6}$ Department of Immunology, College of Medicine, Konkuk University, Chungju, Korea. ${ }^{7}$ Radiation Oncology Branch, Center for Cancer Research, National Cancer Institute, National Institutes of Health, Bethesda, MD, USA.

Received: 27 September 2014 Accepted: 11 December 2014 Published: 15 December 2014

\section{References}

1. Sankaranarayanan R, Ferlay J: Worldwide burden of gynaecological cancer: the size of the problem. Best Pract Res Clin Obstet Gynaecol 2006, 20(2):207-225

2. Clifford GM, Smith JS, Plummer M, Munoz N, Franceschi S: Human papillomavirus types in invasive cervical cancer worldwide: a meta-analysis. Br J Cancer 2003, 88(1):63-73.

3. Dalstein V, Riethmuller D, Pretet $J$, Le Bail CK, Sautiere $J$, Carbillet JP, Kantelip B, Schaal JP, Mougin C: Persistence and load of high-risk HPV are predictors for development of high-grade cervical lesions: a longitudinal French cohort study. Int J Cancer 2003, 106(3):396-403.

4. Snijders PJ, Steenbergen RD, Heideman DA, Meijer CJ: HPV-mediated cervical carcinogenesis: concepts and clinical implications. J Pathol 2006, 208(2):152-164.

5. Lee WC, Lee SY, Koo YJ, Kim TJ, Hur SY, Hong SR, Kim SS, Kee MK, Rhee JE, Lee JS, Choi HS, Cho CH, Kim KT, Park JS: Establishment of a Korea HPV cohort study. J Gynecol Oncol 2013, 24(1):59-65. 
6. Ahdieh L, Munoz A, Vlahov D, Trimble CL, Timpson LA, Shah K: Cervical neoplasia and repeated positivity of human papillomavirus infection in human immunodeficiency virus-seropositive and -seronegative women. Am J Epidemiol 2000, 151(12):1148-1157.

7. Trinchieri G: Biology of natural killer cells. Adv Immunol 1989, 47:187-376.

8. Ishigami S, Natsugoe S, Tokuda K, Nakajo A, Che X, Iwashige H, Aridome K, Hokita S, Aikou T: Prognostic value of intratumoral natural killer cells in gastric carcinoma. Cancer 2000, 88(3):577-583.

9. Villegas FR, Coca S, Villarrubia VG, Jimenez R, Chillon MJ, Jareno J, Zuil M, Callol L: Prognostic significance of tumor infiltrating natural killer cells subset CD57 in patients with squamous cell lung cancer. Lung Cancer 2002, 35(1):23-28.

10. Garzetti GG, Ciavattini A, Muzzioli M, Goteri G, Mannello B, Romanini C, Fabris N: Natural killer cell activity in patients with invasive cervical carcinoma: importance of a longitudinal evaluation in follow-up. Gynecol Obstet Invest 1995, 40(2):133-138.

11. Raulet DH: Roles of the NKG2D immunoreceptor and its ligands. Nat Rev Immunol 2003, 3(10):781-790.

12. Groh V, Bahram S, Bauer S, Herman A, Beauchamp M, Spies T: Cell stress-regulated human major histocompatibility complex class I gene expressed in gastrointestinal epithelium. Proc Natl Acad Sci U S A 1996, 93(22):12445-12450.

13. Jinushi M, Takehara T, Tatsumi T, Kanto T, Groh V, Spies T, Kimura R, Miyagi T, Mochizuki K, Sasaki Y, Hayashi N: Expression and role of MICA and MICB in human hepatocellular carcinomas and their regulation by retinoic acid. Int J Cancer 2003, 104(3):354-361

14. Textor S, Durst $M$, Jansen $L$, Accardi $R$, Tommasino M, Trunk MJ, Porgador A, Watzl C, Gissmann L, Cerwenka A: Activating NK cell receptor ligands are differentially expressed during progression to cervical cancer. Int J Cancer 2008, 123(10):2343-2353.

15. Castriconi R, Cantoni C, Della Chiesa M, Vitale M, Marcenaro E, Conte R, Biassoni R, Bottino C, Moretta L, Moretta A: Transforming growth factor beta 1 inhibits expression of NKp30 and NKG2D receptors: consequences for the NK-mediated killing of dendritic cells. Proc Natl Acad Sci U S A 2003, 100(7):4120-4125.

16. Pende D, Rivera P, Marcenaro S, Chang CC, Biassoni R, Conte R, Kubin M, Cosman D, Ferrone S, Moretta L, Moretta A: Major histocompatibility complex class I-related chain A and UL16-binding protein expression on tumor cell lines of different histotypes: analysis of tumor susceptibility to NKG2D-dependent natural killer cell cytotoxicity. Cancer Res 2002, 62(21):6178-6186.

17. Therasse P, Arbuck SG, Eisenhauer EA, Wanders J, Kaplan RS, Rubinstein L, Verweij J, Van Glabbeke M, van Oosterom AT, Christian MC, Gwyther SG: New guidelines to evaluate the response to treatment in solid tumors. European Organization for Research and Treatment of Cancer, National Cancer Institute of the United States, National Cancer Institute of Canada. J Natl Cancer Inst 2000, 92(3):205-216.

18. Dunn GP, Bruce AT, Ikeda H, Old $\sqcup$, Schreiber RD: Cancer immunoediting: from immunosurveillance to tumor escape. Nat Immunol 2002, 3(11):991-998.

19. McGilvray RW, Eagle RA, Watson NF, Al-Attar A, Ball G, Jafferii I, Trowsdale J, Durrant LG: NKG2D ligand expression in human colorectal cancer reveals associations with prognosis and evidence for immunoediting. Clin Cancer Res 2009, 15(22):6993-7002.

20. Dunn GP, Old $\amalg$, Schreiber RD: The three Es of cancer immunoediting. Annu Rev Immunol 2004, 22:329-360.

21. Shankaran V, Ikeda H, Bruce AT, White JM, Swanson PE, Old LJ, Schreiber RD: IFNgamma and lymphocytes prevent primary tumour development and shape tumour immunogenicity. Nature 2001, 410(6832):1107-1111.

22. Cerwenka A, Baron JL, Lanier LL: Ectopic expression of retinoic acid early inducible-1 gene (RAE-1) permits natural killer cell-mediated rejection of a MHC class I-bearing tumor in vivo. Proc Natl Acad Sci U S A 2001 98(20):11521-11526.

23. Diefenbach $A$, Jensen $E R$, Jamieson AM, Raulet $\mathrm{DH}$ : Rae1 and $\mathrm{H} 60$ ligands of the NKG2D receptor stimulate tumour immunity. Nature 2001, 413(6852):165-171.

24. Smyth MJ, Swann J, Cretney E, Zerafa N, Yokoyama WM, Hayakawa Y: NKG2D function protects the host from tumor initiation. J Exp Med 2005, 202(5):583-588.

25. Stern-Ginossar N, Gur C, Biton M, Horwitz E, Elboim M, Stanietsky N, Mandelboim M, Mandelboim O: Human microRNAs regulate stress-induced immune responses mediated by the receptor NKG2D. Nat Immunol 2008, 9(9):1065-1073.

26. Gasser S, Orsulic S, Brown EJ, Raulet DH: The DNA damage pathway regulates innate immune system ligands of the NKG2D receptor. Nature 2005, 436(7054):1186-1190.

27. Armeanu S, Bitzer M, Lauer UM, Venturelli S, Pathil A, Krusch M, Kaiser S, Jobst J, Smirnow I, Wagner A, Steinle A, Salih HR: Natural killer cell-mediated lysis of hepatoma cells via specific induction of NKG2D ligands by the histone deacetylase inhibitor sodium valproate. Cancer Res 2005, 65(14):6321-6329.

28. Boissel N, Rea D, Tieng V, Dulphy N, Brun M, Cayuela JM, Rousselot P, Tamouza R, Le Bouteiller P, Mahon FX, Steinle A, Salih HR: BCR/ABL oncogene directly controls MHC class I chain-related molecule A expression in chronic myelogenous leukemia. J Immunol 2006, 176(8):5108-5116.

29. Li K, Mandai M, Hamanishi J, Matsumura N, Suzuki A, Yagi H, Yamaguchi K, Baba T, Fujii S, Konishi I: Clinical significance of the NKG2D ligands, MICA/ $B$ and ULBP2 in ovarian cancer: high expression of ULBP2 is an indicator of poor prognosis. Cancer Immunol Immunother 2009, 58(5):641-652.

30. Eagle RA, Jafferji I, Barrow AD: Beyond stressed self: evidence for NKG2D ligand expression on healthy cells. Curr Immunol Rev 2009, 5(1):22-34.

31. Eagle RA, Traherne JA, Ashiru O, Wills MR, Trowsdale J: Regulation of NKG2D ligand gene expression. Hum Immunol 2006, 67(3):159-169.

32. Eagle RA, Trowsdale J: Promiscuity and the single receptor: NKG2D. Nat Rev Immunol 2007, 7(9):737-744.

33. McGilvray RW, Eagle RA, Rolland P, Jafferji I, Trowsdale J, Durrant LG: ULBP2 and RAET1E NKG2D ligands are independent predictors of poor prognosis in ovarian cancer patients. Int J Cancer 2010, 127(6):1412-1420.

34. Cao W, Xi X, Hao Z, Li W, Kong Y, Cui L, Ma C, Ba D, He W: RAET1E2, a soluble isoform of the UL16-binding protein RAET1E produced by tumor cells, inhibits NKG2D-mediated NK cytotoxicity. J Biol Chem 2007, 282(26):18922-18928.

doi:10.1186/1471-2407-14-957

Cite this article as: Cho et al:: MICA/B and ULBP1 NKG2D ligands are independent predictors of good prognosis in cervical cancer. BMC Cancer 2014 14:957.

\section{Submit your next manuscript to BioMed Central and take full advantage of:}

- Convenient online submission

- Thorough peer review

- No space constraints or color figure charges

- Immediate publication on acceptance

- Inclusion in PubMed, CAS, Scopus and Google Scholar

- Research which is freely available for redistribution 